\title{
Polynomially Bounded Sequences and Polynomial Sequences
}

\author{
Hiroyuki Okazaki \\ Shinshu University \\ Nagano, Japan
}

\author{
Yuichi Futa \\ Japan Advanced Institute \\ of Science and Technology \\ Ishikawa, Japan
}

\begin{abstract}
Summary. In this article, we formalize polynomially bounded sequences that plays an important role in computational complexity theory. Class $\mathrm{P}$ is a fundamental computational complexity class that contains all polynomial-time decision problems [11, 12. It takes polynomially bounded amount of computation time to solve polynomial-time decision problems by the deterministic Turing machine. Moreover we formalize polynomial sequences [5].
\end{abstract}

MSC: 03D15 68Q15 03B35

Keywords: computational complexity; polynomial time

MML identifier: ASYMPT_2, version: 8.1.04 5.32.1246

The notation and terminology used in this paper have been introduced in the following articles: [26], [18, [16], 17, 6], 22], 10], 7], 8], 24, 14, 19, 2], 3], [13], 20], 27], [28], 21], [25, and [9].

\section{PReliminaries}

Now we state the proposition:

(1) Let us consider natural numbers $m, k$. If $1 \leqslant m$, then $1 \leqslant m^{k}$.

Let us consider natural numbers $m, n$. Now we state the propositions:

(2) $m \leqslant m^{n+1}$.

(3) If $2 \leqslant m$, then $n+1 \leqslant m^{n}$. 
(4) Let us consider a natural number $k$. Then $2 \cdot k \leqslant 2^{k}$.

Proof: Define $\mathcal{P}$ [natural number] $\equiv 2 \cdot \$_{1} \leqslant 2^{\$_{1}}$. For every natural number $n$ such that $\mathcal{P}[n]$ holds $\mathcal{P}[n+1]$ by [20, (25)], [24, (5)], [1, (14)], (2). For every natural number $n, \mathcal{P}[n]$ from [1, Sch. 2].

(5) Let us consider natural numbers $k, n$. If $k \leqslant n$, then $n+k \leqslant 2^{n}$.

Proof: Define $\mathcal{P}$ [natural number] $\equiv \$_{1}+k+k \leqslant 2^{\$_{1}+k} .2 \cdot k \leqslant 2^{k}$. For every natural number $n$ such that $\mathcal{P}[n]$ holds $\mathcal{P}[n+1]$ by $[20,(27),(25)$, (24)]. For every natural number $n, \mathcal{P}[n]$ from [1, Sch. 2].

(6) Let us consider natural numbers $k, m$. If $2 \cdot k+1 \leqslant m$, then $2^{k} \leqslant 2^{m} / m$. The theorem is a consequence of (5).

(7) Let us consider real numbers $a, b, c$. If $1<a$ and $0<b \leqslant c$, then $\log _{a} b \leqslant \log _{a} c$.

Let us consider a natural number $n$ and a real number $a$. Now we state the propositions:

(8) If $1<a$, then $a^{n}<a^{n+1}$.

(9) If $1 \leqslant a$, then $a^{n} \leqslant a^{n+1}$.

(10) There exists a partial function $g$ from $\mathbb{R}$ to $\mathbb{R}$ such that

(i) $\operatorname{dom} g=] 0,+\infty[$, and

(ii) for every real number $x$ such that $x \in] 0,+\infty\left[\right.$ holds $g(x)=\log _{2} x$, and

(iii) $g$ is differentiable on $] 0,+\infty[$, and

(iv) for every real number $x$ such that $x \in] 0,+\infty[$ holds $g$ is differentiable in $x$ and $g^{\prime}(x)=\log _{2} e / x$ and $0<g^{\prime}(x)$.

Proof: Set $g=\log _{2} e \cdot($ the function $\ln$ ). For every real number $d$ such that $d \in] 0,+\infty\left[\right.$ holds $g(d)=\log _{2} d$ by [20, (56)]. For every real number $x$ such that $x \in] 0,+\infty$ [ holds $g$ is differentiable in $x$ and $g^{\prime}(x)=\log _{2} e / x$ and $0<g^{\prime}(x)$ by [23, (18)], [22, (15)], [20, (57)], [23, (11)].

(11) There exists a partial function $f$ from $\mathbb{R}$ to $\mathbb{R}$ such that

(i) $] e,+\infty[=\operatorname{dom} f$, and

(ii) for every real number $x$ such that $x \in \operatorname{dom} f$ holds $f(x)=x / \log _{2} x$, and

(iii) $f$ is differentiable on $] e,+\infty[$, and

(iv) for every real number $x_{0}$ such that $\left.x_{0} \in\right] e,+\infty\left[\right.$ holds $0 \leqslant f^{\prime}\left(x_{0}\right)$, and

(v) $f$ is non-decreasing. 
Proof: Consider $g$ being a partial function from $\mathbb{R}$ to $\mathbb{R}$ such that $\operatorname{dom} g=$ ] $0,+\infty[$ and for every real number $x$ such that $x \in] 0,+\infty[$ holds $g(x)=$ $\log _{2} x$ and $g$ is differentiable on $] 0,+\infty[$ and for every real number $x$ such that $x \in] 0,+\infty$ [ holds $g$ is differentiable in $x$ and $g^{\prime}(x)=\log _{2} e / x$ and $0<g^{\prime}(x)$. Set $\left.g_{0}=g \uparrow\right] e,+\infty[$. For every object $x$ such that $x \in] e,+\infty[$ holds $x \in] 0,+\infty\left[\right.$ by [23, (11)]. Set $f=\mathrm{id}_{\Omega_{\mathbb{R}}} / g_{0} \cdot g_{0}^{-1}(\{0\})=\emptyset$ by $[23$, (11)], [7, (49)], [4, (10)], [20, (52)]. For every real number $x$ such that $x \in$ dom $f$ holds $f(x)=x / \log _{2} x$ by [7, (49)]. For every real number $x$ such that $x \in] e,+\infty\left[\right.$ holds $f$ is differentiable in $x$ and $f^{\prime}(x)=\log _{2} x-\log _{2} e /\left(\log _{2} x\right)^{2}$ by [23, (11)], [7, (49)], [4, (10)], [20, (52)]. For every real number $x$ such that $x \in] e,+\infty\left[\right.$ holds $0 \leqslant f^{\prime}(x)$ by [20, (57)], [23, (11)].

(12) Let us consider real numbers $x, y$. If $e<x \leqslant y$, then $x / \log _{2} x \leqslant y / \log _{2} y$. The theorem is a consequence of (11).

(13) Let us consider a natural number $k$. Suppose $e<k$. Then there exists a natural number $N$ such that for every natural number $n$ such that $N \leqslant n$ holds $2^{k} \leqslant n / \log _{2} n$. The theorem is a consequence of (12) and (6).

Let us consider a natural number $x$. Let us assume that $1<x$.

(14) There exists a natural number $N$ such that for every natural number $n$ such that $N \leqslant n$ holds $4<n / \log _{x} n$.

(15) There exist natural numbers $N, c$ such that for every natural number $n$ such that $N \leqslant n$ holds $n^{x} \leqslant c \cdot x^{n}$.

(16) Let us consider a natural number $x$. Suppose $1<x$. Then there exist no natural numbers $N, c$ such that for every natural number $n$ such that $N \leqslant n$ holds $2^{n} \leqslant c \cdot n^{x}$.

Proof: Consider $N$ being a natural number such that there exists a natural number $c$ such that for every natural number $n$ such that $N \leqslant n$ holds $2^{n} \leqslant c \cdot n^{x} . N \neq 0$ by [20, (42), (24)]. Consider $c$ being a natural number such that for every natural number $n$ such that $N \leqslant n$ holds $2^{n} \leqslant c \cdot n^{x}$. There exists an element $n$ of $\mathbb{N}$ such that $N \leqslant n$ and $0<n-(x / 4)$ by [24, (6), (3)]. Consider $n$ being an element of $\mathbb{N}$ such that $N \leqslant n$ and $0<n-(x / 4)$. $0<c$ by [20, (34)]. For every natural number $k$ such that $1 \leqslant k$ holds $2^{k \cdot n} \leqslant c \cdot(k \cdot n)^{x}$. For every natural number $k$ such that $1 \leqslant k$ holds $k \cdot n \leqslant \log _{2} c+x \cdot \log _{2} k+x \cdot \log _{2} n$ by [20, (34)], (7), [20, (55), (52), (53)]. Consider $Z$ being an element of $\mathbb{N}$ such that for every natural number $k$ such that $Z \leqslant k$ holds $4<k / \log _{2} k$. There exists a natural number $k$ such that $Z \leqslant k$ and $\log _{2} c+x \cdot \log _{2} n / n-(x / 4) \leqslant k$ by [24, (6), (3)]. There exists a natural number $k$ such that $Z \leqslant k$ and $\log _{2} c+x \cdot \log _{2} n / n-(x / 4) \leqslant k$ and $1<k$ by [1, (11)]. Consider $k$ being a natural number such that $Z \leqslant k$ and $1<k$ and $\log _{2} c+x \cdot \log _{2} n / n-(x / 4) \leqslant k$. 
(17) Let us consider natural numbers $a$, b. If $a \leqslant b$, then $\left\{n^{a}\right\}_{n \in \mathbb{N}} \in O\left(\left\{n^{b}\right\}_{n \in \mathbb{N}}\right)$.

(18) Let us consider a natural number $x$. Suppose $1<x$. Then there exist no natural numbers $N, c$ such that for every natural number $n$ such that $N \leqslant n$ holds $x^{n} \leqslant c \cdot n^{x}$.

Proof: There exist natural numbers $N, c$ such that for every natural number $n$ such that $N \leqslant n$ holds $2^{n} \leqslant c \cdot n^{x}$ by [24, (7)].

(19) Let us consider a non negative real number $a$, and a natural number $n$. If $1 \leqslant n$, then $0<\left\{n^{a}\right\}_{n \in \mathbb{N}}(n)$.

\section{Polynomially Bounded Sequences}

Let $p$ be a sequence of real numbers. We say that $p$ is polynomially bounded if and only if

(Def. 1) there exists a natural number $k$ such that $p \in O\left(\left\{n^{k}\right\}_{n \in \mathbb{N}}\right)$.

Now we state the propositions:

(20) Let us consider a sequence $f$ of real numbers. Suppose $f$ is not polynomially bounded. Let us consider a natural number $k$. Then $f \notin O\left(\left\{n^{k}\right\}_{n \in \mathbb{N}}\right)$.

(21) Let us consider a sequence $f$ of real numbers. Suppose for every natural number $k, f \notin O\left(\left\{n^{k}\right\}_{n \in \mathbb{N}}\right)$. Then $f$ is not polynomially bounded.

(22) Let us consider a positive real number $a$. Then $\left\{a^{1 \cdot n+0}\right\}_{n \in \mathbb{N}}$ is positive.

Let us consider a real number $a$. Now we state the propositions:

(23) If $1 \leqslant a$, then $\left\{a^{1 \cdot n+0}\right\}_{n \in \mathbb{N}}$ is non-decreasing. The theorem is a consequence of (9).

(24) If $1<a$, then $\left\{a^{1 \cdot n+0}\right\}_{n \in \mathbb{N}}$ is increasing. The theorem is a consequence of $(8)$.

(25) Let us consider a natural number $a$. If $1<a$, then $\left\{a^{1 \cdot n+0}\right\}_{n \in \mathbb{N}}$ is not polynomially bounded.

Proof: Consider $k$ being a natural number such that $\left\{a^{1 \cdot n+0}\right\}_{n \in \mathbb{N}} \in$ $O\left(\left\{n^{k}\right\}_{n \in \mathbb{N}}\right)$. Reconsider $f=\left\{n^{k}\right\}_{n \in \mathbb{N}}$ as an eventually positive sequence of real numbers. Reconsider $t=\left\{a^{1 \cdot n+0}\right\}_{n \in \mathbb{N}}$ as an eventually nonnegative sequence of real numbers. $t \in O(f)$ and for every element $n$ of $\mathbb{N}$ such that $1 \leqslant n$ holds $0<f(n)$. Consider $c$ being a real number such that $c>0$ and for every element $n$ of $\mathbb{N}$ such that $n \geqslant 1$ holds $\left(\left\{a^{1 \cdot n+0}\right\}_{n \in \mathbb{N}}\right)(n) \leqslant c \cdot\left\{n^{k}\right\}_{n \in \mathbb{N}}(n)$. For every natural number $n$ such that $n \geqslant 1$ holds $2^{n} \leqslant c \cdot n^{k}$ by [24, (7)]. There exist natural numbers $N, b$ such that for every natural number $n$ such that $N \leqslant n$ holds $2^{n} \leqslant b \cdot n^{k}$ by 24 , $(3)]$. 


\section{Polynomial Sequences}

Now we state the proposition:

(26) Let us consider a finite 0 -sequence $x$ of $\mathbb{R}$, and a sequence $y$ of real numbers. Then

(i) $x \cdot y$ is a finite transfinite sequence of elements of $\mathbb{R}$, and

(ii) $\operatorname{dom}(x \cdot y)=\operatorname{dom} x$, and

(iii) for every object $i$ such that $i \in \operatorname{dom} x$ holds $(x \cdot y)(i)=x(i) \cdot y(i)$.

Let $x$ be a finite 0 -sequence of $\mathbb{R}$ and $y$ be a sequence of real numbers. Observe that the functor $x \cdot y$ yields a finite 0 -sequence of $\mathbb{R}$. Now we state the proposition:

(27) Let us consider a finite 0 -sequence $d$ of $\mathbb{R}$, and natural numbers $x, i$. Suppose $i \in \operatorname{dom} d$. Then $\left(d \cdot\left\{x^{1 \cdot n+0}\right\}_{n \in \mathbb{N}}\right)(i)=d(i) \cdot x^{i}$. The theorem is a consequence of (26).

Let $c$ be a finite 0 -sequence of $\mathbb{R}$. The functor $\operatorname{Seq}_{\text {poly }}(c)$ yielding a sequence of real numbers is defined by

(Def. 2) for every natural number $x, i t(x)=\sum\left(c \cdot\left\{x^{1 \cdot n+0}\right\}_{n \in \mathbb{N}}\right)$.

Let us consider a finite 0 -sequence $d$ of $\mathbb{R}$ and a natural number $k$. Now we state the propositions:

(28) Suppose len $d=k+1$. Then there exists a real number $a$ and there exists a finite 0 -sequence $d_{1}$ of $\mathbb{R}$ and there exists a sequence $y$ of real numbers such that len $d_{1}=k$ and $d_{1}=d\left\lceil k\right.$ and $a=d(k)$ and $d=d_{1} \frown\langle a\rangle$ and $\operatorname{Seq}_{\text {poly }}(d)=\operatorname{Seq}_{\text {poly }}\left(d_{1}\right)+y$ and for every natural number $i, y(i)=a \cdot i^{k}$. PROOF: Consider $a$ being a real number, $d_{1}$ being a finite 0 -sequence of $\mathbb{R}$ such that len $d_{1}=k$ and $d_{1}=d\left\lceil k\right.$ and $a=d(k)$ and $d=d_{1} \frown\langle a\rangle$. Define $\mathcal{F}$ (natural number) $=a \cdot \$_{1}^{k}$. Consider $y$ being a sequence of real numbers such that for every natural number $x, y(x)=\mathcal{F}(x)$ from [15, Sch. 1]. For every element $x$ of $\mathbb{N},\left(\operatorname{Seq}_{\text {poly }}(d)\right)(x)=\left(\operatorname{Seq}_{\text {poly }}\left(d_{1}\right)+y\right)(x)$ by $(26),[1$, (13), (44)], (27).

(29) If len $d=1$, then there exists a real number $a$ such that $a=d(0)$ and for every natural number $x,\left(\operatorname{Seq}_{\text {poly }}(d)\right)(x)=a$. The theorem is a consequence of (26).

(30) If len $d=1$ and $d$ is non-negative yielding, then $\operatorname{Seq}_{\text {poly }}(d) \in O\left(\left\{n^{k}\right\}_{n \in \mathbb{N}}\right)$. The theorem is a consequence of (29).

(31) Let us consider a natural number $k$, a real number $a$, and a sequence $y$ of real numbers. Suppose $0 \leqslant a$ and for every natural number $i, y(i)=a \cdot i^{k}$. Then $y \in O\left(\left\{n^{k}\right\}_{n \in \mathbb{N}}\right)$. 
(32) Let us consider natural numbers $k$, $n$. If $k \leqslant n$, then $O\left(\left\{n^{k}\right\}_{n \in \mathbb{N}}\right) \subseteq$ $O\left(\left\{n^{n}\right\}_{n \in \mathbb{N}}\right)$.

Proof: Consider $i$ being a natural number such that $n=k+i$. Define $\mathcal{P}$ [natural number] $\equiv O\left(\left\{n^{k}\right\}_{n \in \mathbb{N}}\right) \subseteq O\left(\left\{n^{\left(k+\$_{1}\right)}\right\}_{n \in \mathbb{N}}\right)$. For every natural number $x$ such that $\mathcal{P}[x]$ holds $\mathcal{P}[x+1]$. For every natural number $x, \mathcal{P}[x]$ from [1, Sch. 2].

(33) Let us consider a natural number $k$, and a non-negative yielding finite 0 -sequence $c$ of $\mathbb{R}$. Suppose len $c=k+1$. Then $\operatorname{Seq}_{\text {poly }}(c) \in O\left(\left\{n^{k}\right\}_{n \in \mathbb{N}}\right)$. Proof: Define $\mathcal{P}$ [natural number $] \equiv$ for every non-negative yielding finite 0 -sequence $c$ of $\mathbb{R}$ such that len $c=\$_{1}+1$ holds $\operatorname{Seq}_{\text {poly }}(c) \in O\left(\left\{n^{\$_{1}}\right\}_{n \in \mathbb{N}}\right)$. $\mathcal{P}[0]$. For every natural number $k$ such that $\mathcal{P}[k]$ holds $\mathcal{P}[k+1]$ by $(28)$, [7. (47)], [1, (13), (39)]. For every natural number $k, \mathcal{P}[k]$ from [1, Sch. 2].

(34) Let us consider a natural number $k$, and a finite 0 -sequence $c$ of $\mathbb{R}$. Then there exists a finite 0 -sequence $d$ of $\mathbb{R}$ such that

(i) len $d=$ len $c$, and

(ii) for every natural number $i$ such that $i \in \operatorname{dom} d$ holds $d(i)=|c(i)|$.

Proof: Define $\mathcal{F}$ (natural number) $=\left|c\left(\$_{1}\right)\right|(\in \mathbb{R})$. Consider $d$ being a finite 0 -sequence of $\mathbb{R}$ such that len $d=\operatorname{len} c$ and for every natural number $j$ such that $j \in$ len $c$ holds $d(j)=\mathcal{F}(j)$ from [18, Sch. 1].

(35) Let us consider a finite 0 -sequence $c$ of $\mathbb{R}$, and a finite 0 -sequence $d$ of $\mathbb{R}$. Suppose len $d=$ len $c$ and for every natural number $i$ such that $i \in \operatorname{dom} d$ holds $d(i)=|c(i)|$. Let us consider a natural number $n$. Then $\left(\operatorname{Seq}_{\text {poly }}(c)\right)(n) \leqslant\left(\operatorname{Seq}_{\text {poly }}(d)\right)(n)$.

ProOF: $\operatorname{dom}\left(d \cdot\left\{x^{1 \cdot n+0}\right\}_{n \in \mathbb{N}}\right)=\operatorname{dom} d$. For every natural number $i$ such that $i \in \operatorname{dom}\left(c \cdot\left\{x^{1 \cdot n+0}\right\}_{n \in \mathbb{N}}\right)$ holds $\left(c \cdot\left\{x^{1 \cdot n+0}\right\}_{n \in \mathbb{N}}\right)(i) \leqslant\left(d \cdot\left\{x^{1 \cdot n+0}\right\}_{n \in \mathbb{N}}\right)(i)$ by $(26),(27),[19,(4)]$.

(36) Let us consider a natural number $k$, and a finite 0 -sequence $c$ of $\mathbb{R}$. Suppose len $c=k+1$ and $\operatorname{Seq}_{\text {poly }}(c)$ is eventually nonnegative. Then $\operatorname{Seq}_{\text {poly }}(c) \in O\left(\left\{n^{k}\right\}_{n \in \mathbb{N}}\right)$.

ProOF: Consider $d$ being a finite 0 -sequence of $\mathbb{R}$ such that len $d=\operatorname{len} c$ and for every natural number $i$ such that $i \in \operatorname{dom} d$ holds $d(i)=|c(i)|$. For every natural number $i$ such that $i \in \operatorname{dom} d$ holds $0 \leqslant d(i)$ by $[6$, (46)]. For every real number $r$ such that $r \in \operatorname{rng} d$ holds $0 \leqslant r$. $\operatorname{Seq}_{\text {poly }}(d) \in$ $O\left(\left\{n^{k}\right\}_{n \in \mathbb{N}}\right)$. Consider $t$ being an element of $\mathbb{R}^{\mathbb{N}}$ such that $\operatorname{Seq}_{\text {poly }}(d)=t$ and there exists a real number $c$ and there exists an element $N$ of $\mathbb{N}$ such that $c>0$ and for every element $n$ of $\mathbb{N}$ such that $n \geqslant N$ holds $t(n) \leqslant$ $c \cdot\left\{n^{k}\right\}_{n \in \mathbb{N}}(n)$ and $t(n) \geqslant 0$. Consider $N_{1}$ being a natural number such that for every natural number $n$ such that $N_{1} \leqslant n$ holds $0 \leqslant\left(\operatorname{Seq}_{\text {poly }}(c)\right)(n)$. 
Consider $a$ being a real number, $N_{2}$ being an element of $\mathbb{N}$ such that $a>0$ and for every element $n$ of $\mathbb{N}$ such that $n \geqslant N_{2}$ holds $t(n) \leqslant a \cdot\left\{n^{k}\right\}_{n \in \mathbb{N}}(n)$ and $t(n) \geqslant 0$. Set $N=N_{1}+N_{2}$. For every element $n$ of $\mathbb{N}$ such that $n \geqslant N$ holds $\left(\operatorname{Seq}_{\text {poly }}(c)\right)(n) \leqslant a \cdot\left\{n^{k}\right\}_{n \in \mathbb{N}}(n)$ and $\left(\operatorname{Seq}_{\text {poly }}(c)\right)(n) \geqslant 0$ by [1, (11)], $(35)$.

(37) Let us consider natural numbers $k, n$. If $0<n$, then $n \cdot\left\{n^{k}\right\}_{n \in \mathbb{N}}(n)=$ $\left\{n^{(k+1)}\right\}_{n \in \mathbb{N}}(n)$.

(38) Let us consider a finite 0 -sequence $c$ of $\mathbb{R}$. Suppose len $c=0$. Let us consider a natural number $x$. Then $\left(\operatorname{Seq}_{\text {poly }}(c)\right)(x)=0$.

(39) Let us consider an eventually nonnegative sequence $f$ of real numbers, and a natural number $k$. Suppose $f \in O\left(\left\{n^{k}\right\}_{n \in \mathbb{N}}\right)$. Then there exists a natural number $N$ such that for every natural number $n$ such that $N \leqslant n$ holds $f(n) \leqslant\left\{n^{(k+1)}\right\}_{n \in \mathbb{N}}(n)$. The theorem is a consequence of $(37)$.

(40) Let us consider a finite 0 -sequence $c$ of $\mathbb{R}$. Then there exists a finite 0 -sequence $a_{1}$ of $\mathbb{R}$ such that

(i) $a_{1}=|c|$, and

(ii) for every natural number $n,\left(\operatorname{Seq}_{\text {poly }}(c)\right)(n) \leqslant\left(\operatorname{Seq}_{\text {poly }}\left(a_{1}\right)\right)(n)$.

Proof: Reconsider $a_{1}=|c|$ as a finite 0 -sequence of $\mathbb{R}$. Set $m_{1}=c$. $\left\{n^{1 \cdot n+0}\right\}_{n \in \mathbb{N}}$. Set $m_{2}=a_{1} \cdot\left\{n^{1 \cdot n+0}\right\}_{n \in \mathbb{N}}$. For every natural number $x$ such that $x \in \operatorname{dom} m_{1}$ holds $m_{1}(x) \leqslant m_{2}(x)$ by [19, (4)].

(41) Let us consider finite 0 -sequences $c, a_{1}$ of $\mathbb{R}$. Suppose $a_{1}=|c|$. Let us consider a natural number $n$. Then $\left|\left(\operatorname{Seq}_{\text {poly }}(c)\right)(n)\right| \leqslant\left(\operatorname{Seq}_{\text {poly }}\left(a_{1}\right)\right)(n)$.

Proof: Define $\mathcal{P}$ [natural number] $\equiv$ for every finite 0 -sequences $c, a_{1}$ of $\mathbb{R}$ such that len $c=\$_{1}$ and $a_{1}=|c|$ for every natural number $x$, $\left|\left(\operatorname{Seq}_{\text {poly }}(c)\right)(x)\right| \leqslant\left(\operatorname{Seq}_{\text {poly }}\left(a_{1}\right)\right)(x) \cdot \mathcal{P}[0]$ by $(26)$, 6, (44)]. For every natural number $k$ such that $\mathcal{P}[k]$ holds $\mathcal{P}[k+1]$ by $(28)$, [7, (47)], [15, (7)], [6. (56), (65)]. For every natural number $n, \mathcal{P}[n]$ from [1, Sch. 2].

(42) Let us consider a real number $a$. Suppose $0<a$. Let us consider a natural number $k$, and a non-negative yielding finite 0 -sequence $d$ of $\mathbb{R}$. Suppose len $d=k$. Then there exists a natural number $N$ such that for every natural number $x$ such that $N \leqslant x$ for every natural number $i$ such that $i \in \operatorname{dom} d$ holds $d(i) \cdot x^{i} \cdot k \leqslant a \cdot x^{k}$.

Proof: For every natural number $i$ such that $i \in \operatorname{dom} d$ holds $0 \leqslant d(i)$ by [7. (3)].

(43) Let us consider a natural number $k$, a finite 0 -sequence $d$ of $\mathbb{R}$, a real number $a$, and a sequence $y$ of real numbers. Suppose $0<a$ and len $d=k$ and for every natural number $x, y(x)=a \cdot x^{k}$. Then there exists a natural number $N$ such that for every natural number $x$ such that $N \leqslant x$ holds 
$\left|\left(\operatorname{Seq}_{\text {poly }}(d)\right)(x)\right| \leqslant y(x)$. The theorem is a consequence of $(38),(42),(26)$, (27), and (41).

(44) Let us consider a natural number $k$, and a finite 0 -sequence $d$ of $\mathbb{R}$. Suppose len $d=k+1$ and $0<d(k)$. Then $\operatorname{Seq}_{\text {poly }}(d)$ is eventually nonnegative. Proof: Consider $a$ being a real number, $d_{1}$ being a finite 0 -sequence of $\mathbb{R}, y$ being a sequence of real numbers such that len $d_{1}=k$ and $d_{1}=d\lceil k$ and $a=d(k)$ and $d=d_{1} \frown\langle a\rangle$ and $\operatorname{Seq}_{\text {poly }}(d)=\operatorname{Seq}_{\text {poly }}\left(d_{1}\right)+y$ and for every natural number $i, y(i)=a \cdot i^{k}$. Consider $N$ being a natural number such that for every natural number $i$ such that $N \leqslant i$ holds $\left|\left(\operatorname{Seq}_{\text {poly }}\left(d_{1}\right)\right)(i)\right| \leqslant y(i)$. For every natural number $i$ such that $N \leqslant i$ holds $0 \leqslant\left(\operatorname{Seq}_{\text {poly }}(d)\right)(i)$ by [19, (4)], [15, (7)].

Let us consider a natural number $k$ and a finite 0 -sequence $c$ of $\mathbb{R}$.

Let us assume that len $c=k+1$ and $0<c(k)$. Now we state the propositions:

(45) $\operatorname{Seq}_{\text {poly }}(c) \in O\left(\left\{n^{k}\right\}_{n \in \mathbb{N}}\right)$.

(46) $\operatorname{Seq}_{\text {poly }}(c)$ is polynomially bounded. The theorem is a consequence of (36) and (44).

ACKNOWLEDGEMENT: The authors would also like to express their gratitude to Prof. Yasunari Shidama for his support and encouragement.

\section{REFERENCES}

[1] Grzegorz Bancerek. The fundamental properties of natural numbers Formalized Mathematics, 1(1):41-46, 1990 .

[2] Grzegorz Bancerek. The ordinal numbers. Formalized Mathematics, 1(1):91-96, 1990.

[3] Grzegorz Bancerek. Increasing and continuous ordinal sequences Formalized Mathematics, 1(4):711-714, 1990.

[4] Grzegorz Bancerek and Piotr Rudnicki. Two programs for SCM. Part I - preliminaries Formalized Mathematics, 4(1):69-72, 1993.

[5] E.J. Barbeau. Polynomials. Springer, 2003.

[6] Czesław Byliński. The complex numbers. Formalized Mathematics, 1(3):507-513, 1990.

[7] Czesław Byliński. Functions and their basic properties. Formalized Mathematics, 1(1): 55-65, 1990.

[8] Czesław Byliński. Functions from a set to a set Formalized Mathematics, 1(1):153-164, 1990.

[9] Czesław Byliński. Some basic properties of sets Formalized Mathematics, 1(1):47-53, 1990.

[10] Agata Darmochwał. Finite sets, Formalized Mathematics, 1(1):165-167, 1990.

[11] Jon Kleinberg and Eva Tardos. Algorithm Design. Addison-Wesley, 2005.

[12] Donald E. Knuth. The Art of Computer Programming, Volume 1: Fundamental Algorithms, Third Edition. Addison-Wesley, 1997.

[13] Artur Korniłowicz. On the real valued functions. Formalized Mathematics, 13(1):181-187, 2005.

[14] Jarosław Kotowicz. The limit of a real function at infinity Formalized Mathematics, 2 (1):17-28, 1991. 
[15] Jarosław Kotowicz. Real sequences and basic operations on them Formalized Mathematics, 1(2):269-272, 1990.

[16] Richard Krueger, Piotr Rudnicki, and Paul Shelley. Asymptotic notation. Part I: Theory Formalized Mathematics, 9(1):135-142, 2001.

[17] Richard Krueger, Piotr Rudnicki, and Paul Shelley. Asymptotic notation. Part II: Examples and problems. Formalized Mathematics, 9(1):143-154, 2001.

[18] Yatsuka Nakamura and Hisashi Ito. Basic properties and concept of selected subsequence of zero based finite sequences. Formalized Mathematics, 16(3):283-288, 2008. doi: $10.2478 /$ v10037-008-0034-y

[19] Jan Popiołek. Some properties of functions modul and signum. Formalized Mathematics, $1(\mathbf{2}): 263-264,1990$.

[20] Konrad Raczkowski and Andrzej Nędzusiak. Real exponents and logarithms Formalized Mathematics, 2(2):213-216, 1991.

[21] Konrad Raczkowski and Andrzej Nędzusiak. Series. Formalized Mathematics, 2(4):449452, 1991.

[22] Konrad Raczkowski and Paweł Sadowski. Real function differentiability Formalized Mathematics, 1(4):797-801, 1990.

[23] Yasunari Shidama. The Taylor expansions Formalized Mathematics, 12(2):195-200, 2004.

[24] Michał J. Trybulec. Integers Formalized Mathematics, 1(3):501-505, 1990.

[25] Zinaida Trybulec. Properties of subsets Formalized Mathematics, 1(1):67-71, 1990.

[26] Tetsuya Tsunetou, Grzegorz Bancerek, and Yatsuka Nakamura. Zero-based finite sequences Formalized Mathematics, 9(4):825-829, 2001.

[27] Edmund Woronowicz. Relations and their basic properties. Formalized Mathematics, 1 (1):73-83, 1990.

[28] Edmund Woronowicz. Relations defined on sets. Formalized Mathematics, 1(1):181-186, 1990. 